\title{
25 Research Soure \\ Fertility and Progeny Ploidy of Triploids in China Rosa Germplasm
}

Hui Feng ( $\square$ fenghui918@gmail.com )

Beijing Institute of Landscape Architecture https://orcid.org/0000-0002-1029-5194

Naizhe Ji

Beijing Institute of Landscape Architecture

Yu Xie

Beijing Institute of landscape Architecture

Beibei Ge

Beijing Institute of Landscape Architecture

Yanhua Bu

Beijing Institute of Landscape Architecture

Shiwei Zhao

Beijing Institute of Landscape Architecture

Research article

Keywords: China Rosa Germplasm, Triploid, Fertility, Progeny Ploidy

Posted Date: October 19th, 2020

DOI: https://doi.org/10.21203/rs.3.rs-68808/v1

License: (c) (1) This work is licensed under a Creative Commons Attribution 4.0 International License. Read Full License 


\section{Abstract \\ Background}

Although triploid plants are often assumed to be sterile, in Rosa, triploids appear to have been important in both species evolution and breeding. A better understanding of the fertility of China Rosa triploids and their behavior when used in interploidy crosses will not only improve gene introgression from China triploid roses to modern roses, but also enhance our understanding of male and female fertility mechanism with triploids. The objective of this study was to evaluate the usefulness of five triploid China roses for breeding by measuring the pollen size, quantifying the fertility and following the ploidy transmission of interploidy crosses.

\section{Results}

In the five triploids, the pollen grain size of Rosa multiflora var. cathayensis Rehd 'Fen Tuan Qiang Wei' followed a normal distribution, suggesting that 1 mainly ploidy level pollen grain were produced, while the pollen size of other 4 triploids followed skewed, flattened, and slightly bimodal distributions, indicating a wide range of chromosome numbers. Although none of the 5 triploids produced hips as females, 3 gave good hip and seed production on the tetraploid mother plant when used as the pollen parents. Based on the ploidy level analysis of their progeny, 'Fen Tuan Qiang Wei' produced $1 \mathrm{n}$ viable pollen whereas 'Chun Shui Lü Bo' and 'Yu Shi Zhuang' produced $2 \mathrm{n}$ viable pollen. In the meiosis of triploids, the triads, pentads and microcyte in the meiosis products indicated the objective triploid production of euploid pollen grains.

\section{Conclusions}

In this study we reported 1 China triploid rose produces $1 \mathrm{n}$ viable pollen, and 2 China triploid roses produce $2 \mathrm{n}$ viable pollen, and no anueploid progeny was produced. Our results indicate that the triploids contribute significantly to tetraploids formation in the crossing system of Genus Rosa, and it is possible for breeders to create novel rose types with valuable triploids in the future. With the special triploid resources found in this experiment, Genus Rosa will be an excellent material to study the mechanism of triploids in the future.

\section{Background}

Polyploidy (whole-genome duplication, WGD) is a major force in the evolution of higher plants (Simillion et al., 2002; Blanc and Wolfe, 2004). Most natural polyploids are thought to origin from sexual mechanism. As the fertilization of two unreduced gametes (female gamete and male gamete) seems to be an unlikely event, natural tetraploids are most likely to origin in two steps, via a triploid intermediary. Some studies about the mechanism of polyploid origin have been reported (Ramsey and Schemske 1998; Burtonand Husband, 2001; Husband, 2004). Most triploid are assumed to be sterile because their gametes are aneuploid, but some triploids do have fertility in breeding (Zhang, 2009; Wang, 2017; Zhang, 2017). The greater variability for pollen size in triploids had been observed, which reflected different ploidy levels or aneuploid gametes 
(Jacob and Pierret 2000; leus 2005;Crespel et al. 2006; Zlesak, 2009). Irregular chromosome pairing and abnormal meiotic division are the reasons for variations in pollen size and fertility, as has been reported in triploid Populus (Wang et al. 2010) and lily (Zhang, 2017). The aberrations during chromosome pairing, segregation, spindle formation, or cytokinesis might produce triads, dyads or monads, which are probably to produce unbalanced gametes (Bosco et al. 1999; Risso-Pascotto et al. 2005; Wang et al. 2010; Zhang, 2017).

Roses are very valuable ornamental plants around the world and thousands of cultivars have been developed. Most botanical species are diploid, while most commercially valuable modern rose cultivars are tetraploid. The hybridization among European tetraploids and Asian diploids produced modern rose cultivars (Zlesak, 2009). It is most likely that triploid roses have played a very important role in this long process as a ploidy bridge. About eight to eleven wild species had been used in the breeding history of modern commercial roses (Mac Phail and Kevan 2009). There are still quantity of diploid wild species and some triploid germplasm, which have not been introduced into modern rose breeding programs. Inter-specific or intergeneric hybrids can improve not only the qualitative but also quantitative traits of growth vigor or ornamental value (Van Tuyl and Lim 2003; Bao et al. 2012). Interploidy crosses with triploids have been reported, but the ploidy levels of the progeny were not consistent. In the crosses between tetraploid females and triploid males, Leus (2005) reported about 98\% (123/125) tetraploid offspring, but Zlesak (2009) obtained about half triploid (23/43) offspring and half tetraploid offspring (20/43). In the crosses between diploid females and triploid males, Barden got diploid, triploid, and tetraploid offspring (Barden and Zlesak, 2004).

Rosa resources from China, such as Rosa chinensis and Chinese Old Garden Roses, have contributed recurrent flowering and tea scent to modern roses, and played very important roles in the formation history of modern roses. In China Rosa germplasm, there are some triploids that have not been used in breeding program but possess valuable traits. A better understanding of the fertility of China Rosa triploids and their behavior when used in interploidy crosses will not only improve gene introgression from China triploid roses to modern roses, but also enhance our understanding of male and female fertility mechanism with triploids. This study will also help us clarify the 'triploid bridge' in modern roses formation history, and make it possible for breeders to create novel rose types in the future. The objectives of this study were to evaluate the usefulness of triploid China rose for breeding by measuring the pollen size, quantifying the fertility and following the ploidy transmission of interploidy crosses.

\section{Methods}

\section{Plant materials}

Five triploid China roses, including 4 Chinese old garden rose cultivars 'Chun Shui Lü Bo', 'Yu Shi Zhuang', 'Hu Zhong Yue', 'Si Mian Jing' and 1 species R. multiflora var. cathayensis Rehd 'Fen Tuan Qiang Wei' were used as triploid material (Table 1). Eight tetraploid modern roses and one diploid Chinese old garden rose were used as hybrid parents. Crosses between the triploids and tetraploids or diploids were conducted to produce populations with ploidy level segregation. Ploidy level analysis was conducted on the 190 randomly selected seedlings from the hybrid population. 
Table 1

Ploidy and rose class of the parents used in this experiment.

\begin{tabular}{|c|c|c|c|c|c|}
\hline Cultivar name & Classi- fication & $\begin{array}{l}\text { Ploidy } \\
\text { level }\end{array}$ & Cultivar name & $\begin{array}{l}\text { Classi- } \\
\text { fication }\end{array}$ & $\begin{array}{l}\text { Ploidy } \\
\text { level }\end{array}$ \\
\hline Old Blush & $\begin{array}{l}\text { Chinese Old Garden } \\
\text { Rose }\end{array}$ & $2 x$ & Chun Chao & Shrub & $4 x$ \\
\hline $\begin{array}{l}\text { R. multiflora 'Fen Tuan } \\
\text { Qiang Wei' }\end{array}$ & Species & $3 x$ & Queen Elizabeth & Hybrid Tea & $4 x$ \\
\hline Chun Shui Lü Bo & $\begin{array}{l}\text { Chinese Old Garden } \\
\text { Rose }\end{array}$ & $3 x$ & Hongwu Yue & Shrub & $4 x$ \\
\hline Hu Zhong Yue & $\begin{array}{l}\text { Chinese Old Garden } \\
\text { Rose }\end{array}$ & $3 x$ & Carefree Beauty & Shrub & $4 x$ \\
\hline Qing Lian Xue Shi & $\begin{array}{l}\text { Chinese Old Garden } \\
\text { Rose }\end{array}$ & $3 x$ & Gold Mary & Floribunda & $4 x$ \\
\hline Yu Shi Zhuang & $\begin{array}{l}\text { Chinese Old Garden } \\
\text { Rose }\end{array}$ & $3 x$ & Compassion & Climber & $4 x$ \\
\hline Tutti Frutti & Shrub & $4 x$ & $\begin{array}{l}\text { Dee Dee } \\
\text { Bridgewater }\end{array}$ & Climber & $4 x$ \\
\hline
\end{tabular}

Note: Rosa multiflora var. cathayensis 'Fen Tuan Qiang Wei' is described in <Flora of China >, vol.9, Rosaceae, Rosa 60b, 2003. Old Blush, 'Chun Shui Lü Bo', 'Hu Zhong Yue', 'Qing Lian Xue Shi', 'Yu Shi Zhuang' are all described in <China Old Garden Rose>, published by China Science Press, 2015. The other modern roses are all described in <Modern Rose $\varangle>$ (2013). All the plant material are commercial available. The authors bought them and identified them according to the books above, and they are all planted in Beijing Institute of Landscape Architecture now.

\section{Pollen viability and size observation}

Fresh pollen sample was collected (Zlesak, 2009), then stored in refrigerator at $-20^{\circ} \mathrm{C}$. The sample was then mounted in a drop of $1 \%$ aceto-carmine on a microscope slide and viewed under the Olympus microscope (DP74, Japan). Empty and shrunken pollen grains were scored. The diameter of 500 pollen grains were measured using the attached calibrated micrometer of Olympus microscope.

Pollen viability was evaluated using the method proposed by Feng et al. (2017). A pollen grain was considered to have germinated if the pollen tube length was equal to or greater than the pollen diameter. Five microscopic field areas were randomly selected, and 5 plant samples were observed.

\section{Pollen morphological observation}

Pollen morphology was studied with scanning electron microscopy (Hitachi S-3400, Tokyo, Japan) following Zhang's method (Zhang, et al. 2017).

\section{Crossing experiment}


Interploidy crosses were conducted by hand in May 2013-2016 as proposed by Gao (2018), and hips were collected in October. Seeds were placed in wet sand storage at $1-4{ }^{\circ} \mathrm{C}$ for $2-3$ months, after which they were sown in a greenhouse at $25^{\circ} \mathrm{C}$. $\mathrm{F}_{1}$ seedlings were planted in an open field. The plant materials in the experiment were collected from and approved by the Beijing Institute of Landscape Architecture.

\section{Observation of Pollen Tube Growth}

Pollinated pistils of Rosa 'Carefree Beauty' were excised $8 \mathrm{~h}, 12 \mathrm{~h}, 24 \mathrm{~h}, 48 \mathrm{~h}$ and $72 \mathrm{~h}$ after hand pollination with pollens from triploid 'Chun Shui Lü Bo'. Pollen tube growth was observed according to Michael's protocol (Michael et al, 2015). Visualization was performed with an Olympus epifluorescence microscope (DP74) using an excitation wavelength of $450 / 90 \mathrm{~nm}$ and an emission wave length of $520 \mathrm{~nm}$.

\section{Stigma receptivity observation}

Stigma receptivity was studied by hydrogen peroxide test under microscope in a laboratory according to Monika's protocol (Monika, 2017). The flowers were taken at the first day the petals reveal the stigmatic surface and the observations were recorded 1 hour intervals from 8 a.m. to 8 p.m. The receptivity of stigma was estimated by the bubbling quantity from stigma.

\section{Analysis of ploidy levels}

Counts of somatic chromosome number was done in shoot tip tissue following Wang's method (Wang, 2017). The preparation was observed by using an Olympus DP75 microscope.

The ploidy level of progeny was also determined by flow cytometry (Feng et al., 2017).Samples were analyzed with a BD Company Accuri C6 flow cytometer. 5000-10,000 particles were detected and collected, 'Old Blush' was used as diploid standard. The somatic chromosome numbers of some seedlings were also checked by counting chromosomes of cells from shoot tips as described above.

\section{Cytological observations of microsporogenesis}

Anthers of 'Fen Tuan Qiang Wei' and 'Chun Shui Lü Bo' undergoing meiotic development were collected and immediately fixed in freshly prepared Carnoy's solution (3:1 ethanol-acetic acid) for $24 \mathrm{~h}$ at room temperature and then stored in $70 \%$ ethanol at $4{ }^{\circ} \mathrm{Cuntil}$ analysis. Microscopic observations and image acquisition were conducted as described by Zhang et al. (2017).

\section{Results}

\section{Pollen morphology, size-frequency distribution and viability}

Pollen grains of the five triploids had 3 colporates that annularly distributed in equal space, and extended to the two poles. They were long elliptic shaped, and their outer wall was coarse, microperforate, and decorated with streaks (Fig. 1; N3P4C5 type, see Erdtman, 1971). Some anomalous pollens were found. The pollen grain size of the 5 triploids were analyzed by using 1 diploid and 1 tetraploid as controls (Table 2). According to $\mathrm{P} / \mathrm{E}$ value, compared with 'Old Blush', the five triploids had more long spherical shaped pollens, and less 
extra long spherical shaped pollens. Compared with the tetraploid 'Dee Dee Bridgwater', 'Yu Shi Zhuang' and 'QingliangXueshi' had similar quantity of long spherical shaped pollens, and the other 3 triploids had fewer long spherical shaped pollen.

Table 2

Analysis of rose pollen morphology.

\begin{tabular}{|c|c|c|c|c|c|c|}
\hline \multirow[t]{3}{*}{$\begin{array}{l}\text { Cultivar } \\
\text { name }\end{array}$} & \multirow[t]{3}{*}{$\begin{array}{l}\text { Polar axis } \\
\text { length }(\mu \mathrm{m}) \\
\pm S E\end{array}$} & \multirow[t]{3}{*}{$\begin{array}{l}\text { Equatorial axis } \\
\text { length }(\mu \mathrm{m}) \pm S E\end{array}$} & $\begin{array}{l}\text { Near } \\
\text { spherical } \\
\text { shape }\end{array}$ & $\begin{array}{l}\text { Long } \\
\text { spherical } \\
\text { shape }\end{array}$ & $\begin{array}{l}\text { Extra long } \\
\text { spherical } \\
\text { shape }\end{array}$ & \multirow[t]{3}{*}{$\begin{array}{l}\text { Coefficient } \\
\text { of } \\
\text { variation }\end{array}$} \\
\hline & & & $(P / E=$ & $(P / E=$ & $(P / E>2)$ & \\
\hline & & & $0.75 \bigotimes 1.33)$ & $1.33 \llbracket 2)$ & & \\
\hline Old Blush & $\begin{array}{l}40.23 \pm \\
0.27 \mathrm{~b}\end{array}$ & $19.00 \pm 0.23 c$ & 0 & $16.7 \%$ & $83.3 \%$ & 11.6 \\
\hline $\begin{array}{l}\text { Dee Dee } \\
\text { Bridge } \\
\text { water }\end{array}$ & $\begin{array}{l}42.80 \pm \\
0.37 a\end{array}$ & $30.01 \pm 0.55 a$ & $25.5 \%$ & $72.6 \%$ & $2.0 \%$ & $15.0 \%$ \\
\hline $\begin{array}{l}\text { Fen Tuan } \\
\text { Qiang Wei }\end{array}$ & $\begin{array}{l}41.27 \pm \\
0.32 \mathrm{~b}\end{array}$ & $20.87 \pm 0.19 b$ & $2.0 \%$ & $48.0 \%$ & $50.0 \%$ & $13.3 \%$ \\
\hline $\begin{array}{l}\text { Chun Shui } \\
\text { Lü Bo }\end{array}$ & $\begin{array}{l}36.85 \pm \\
0.41 \mathrm{c}\end{array}$ & $18.93 \pm 0.19 c$ & $2.7 \%$ & $52.2 \%$ & $45.2 \%$ & $19.3 \%$ \\
\hline $\begin{array}{l}\text { Yu Shi } \\
\text { Zhuang }\end{array}$ & $\begin{array}{l}31.38 \pm \\
0.37 \mathrm{~d}\end{array}$ & $18.45 \pm 0.17 c$ & $11.2 \%$ & $72.5 \%$ & $16.4 \%$ & $20.8 \%$ \\
\hline $\begin{array}{l}\text { Qing Lian } \\
\text { Xue Shi }\end{array}$ & $\begin{array}{l}33.24 \pm \\
0.36 \mathrm{~d}\end{array}$ & $18.78 \pm 0.14 c$ & $3.7 \%$ & $75.8 \%$ & $20.6 \%$ & $18.8 \%$ \\
\hline $\begin{array}{l}\text { Hu Zhong } \\
\text { Yue }\end{array}$ & $\begin{array}{l}31.96 \pm \\
1.25 \mathrm{~d}\end{array}$ & $20.45 \pm 0.67 b$ & $23.7 \%$ & $60.5 \%$ & $15.8 \%$ & $24.3 \%$ \\
\hline
\end{tabular}

Multiple comparisons analysis was conducted on the pollen polar axis length of 'Old Blush', 'Dee Dee Bridgewater' and five triploids (Table 2). 'Dee Dee Bridgewater' pollen was the largest, followed by 'Old Blush' and 'Fen Tuan Qiang Wei'. The pollens of other 4 triploids were smaller than 'Old Blush'. The pollen size variation coefficient of diploid 'Old Blush' and tetraploid 'DEE DEE Bridgewater ' was $11.6 \%$ and $15.0 \%$ respectively. The variation coefficient of triploid 'Fen Tuan Qiang Wei' was $13.3 \%$, whereas the variation coefficient of the other 4 triploids were from $18.8 \%$ to $24.3 \%$, higher than the diploid and tetraploid.

Gaussian distribution tests were conducted on frequency distribution with polar axis length of pollen grains (Table 3). Only 'Old Blush', 'Dee Dee Bridgewater ' and 'Fen Tuan Qiang Wei' showed a normal distribution of pollen size, indicating only one ploidy level pollens were produced. The other four triploids had skewed, flattened, and slightly bimodal distributions, indicating the wide range of pollen size in triploid (Fig. 1). Pollen germination rate of 5 triploids on artificial liquid medium were very different, with 'Fen Tuan Qiang Wei' had the highest germination rate of $32.00 \%$, and 'Huzhong Yue' has the smallest germination rate of $4.00 \%$ (Table 4). 
Table 3

Gaussian distribution tests on frequency distribution with pollen grain size.

\begin{tabular}{|lll|}
\hline Cultivar or species & $\begin{array}{l}\text { Ploid } \\
\text { level }\end{array}$ & Gaussian distribution tests \\
\hline Old Blush & $2 x$ & 0.566 \\
\hline Dee Dee Bridgewater & $4 x$ & 0.399 \\
\hline Fen Tuan Qiang Wei & $3 x$ & 0.230 \\
\hline Chun Shui Lü Bo & $3 x$ & 0.091 \\
\hline Yu Shi Zhuang & $3 x$ & $0 *$ \\
\hline Qing Lian Xue Shi & $3 x$ & $0 *$ \\
\hline Hu Zhong Yue & $3 x$ & 0.089 \\
\hline Note: * indicate a significant difference at Pø0.05. \\
\hline
\end{tabular}

Table 4

Pollen germination rates of five triploid roses.

\begin{tabular}{|lll|}
\hline Cultivar or species & Pollen amount/flower & Pollen germination Rate(\%) \\
\hline Chun Shui Lü Bo & +++ & 10.0 \\
\hline Yu Shi Zhuang & ++ & 6.0 \\
\hline Hu Zhong Yue & + & 4.0 \\
\hline Qing Lian Xue Shi' & + & 5.0 \\
\hline Fen Tuan Qiang Wei & ++++ & 32.0 \\
\hline Note: From '++++' to '+' means the amount of pollen from maximum to minimum. \\
\hline tube growth Observation and male fertility of five \\
ds
\end{tabular}

The pollen of 'Chun Shui Lü Bo' on the stigma of 'Carefree Beauty'germinated 8-12 hours after pollination (Fig. 3a, b, c). By 24 hours the pollen tubes grew through the tissue of the stigma and reached the middle or the upper part of the style (Fig. 3d), by 48 hours the pollen tubes reached the lower middle of the style (Fig. 3e), and by 72 hours after pollination, a few pollen tubes reached the ovary (Fig. 3f).

Higher fruit set and number of seeds per hip were observed in interploidy crosses with the tetraploid 'Dee Dee Bridgewater' as a seed parent and triploid 'Chun Shui Lü Bo', 'Yu Shi Zhuang' and 'Fen Tuan Qiang Wei'as pollen parents (Table 5). The cross between diploid 'Old Blush'( $(\mathbb{\nabla})$ and triploid'Fen Tuan Qiang Wei' (『) had lower fruit set rate and germination rate than the cross between tetraploid 'Dee Dee Bridgewater' ( () ) and 
triploid 'Fen Tuan Qiang Wei' (囚). Crosses with triploid 'Qin Lian Xue Shi' and 'Hu Zhong Yue' as pollen parents did not set any hips. 
Table 5

Results of hybridization with rose triploids as male parents.

\begin{tabular}{|c|c|c|c|c|c|c|c|c|c|}
\hline $\begin{array}{l}\text { Seed } \\
\text { parents }\end{array}$ & $\begin{array}{l}\text { Ploidy } \\
\text { level }\end{array}$ & $\begin{array}{l}\text { Pollen } \\
\text { parents }\end{array}$ & $\begin{array}{l}\text { Ploidy } \\
\text { level }\end{array}$ & $\begin{array}{l}\text { No. of } \\
\text { pollinated } \\
\text { flowers }\end{array}$ & $\begin{array}{l}\text { No. } \\
\text { of } \\
\text { hips }\end{array}$ & $\begin{array}{l}\text { Hip } \\
\text { set } \\
(\%)\end{array}$ & $\begin{array}{l}\text { No of } \\
\text { seeds }\end{array}$ & $\begin{array}{l}\text { No of } \\
\text { seedings }\end{array}$ & $\begin{array}{l}\text { Germina- } \\
\text { tion (\%) }\end{array}$ \\
\hline $\begin{array}{l}\text { Dee Dee } \\
\text { Bridgewater }\end{array}$ & $4 x$ & $\begin{array}{l}\text { Chun } \\
\text { Shui Lü } \\
\text { Bo }\end{array}$ & $3 x$ & 40 & 18 & 45 & 275 & 115 & 42 \\
\hline Tutti Frutti & $4 x$ & $\begin{array}{l}\text { Chun } \\
\text { Shui Lü } \\
\text { Bo }\end{array}$ & $3 x$ & 12 & 7 & 58 & 60 & 20 & 33 \\
\hline Chun Chao & $4 x$ & $\begin{array}{l}\text { Chun } \\
\text { Shui Lü } \\
\text { Bo }\end{array}$ & $3 x$ & 43 & 19 & 44 & 111 & 14 & 13 \\
\hline $\begin{array}{l}\text { Queen } \\
\text { Elizabeth }\end{array}$ & $4 x$ & $\begin{array}{l}\text { Chun } \\
\text { Shui Lü } \\
\text { Bo }\end{array}$ & $3 x$ & 25 & 19 & 76 & 140 & 38 & 27 \\
\hline Compassion & $4 x$ & $\begin{array}{l}\text { Chun } \\
\text { Shui Lü } \\
\text { Bo }\end{array}$ & $3 x$ & 22 & 1 & 5 & 3 & 2 & 67 \\
\hline $\begin{array}{l}\text { Carefree } \\
\text { Beauty }\end{array}$ & $4 x$ & $\begin{array}{l}\text { Chun } \\
\text { Shui Lü } \\
\text { Bo }\end{array}$ & $3 x$ & 48 & 14 & 29 & 33 & 13 & 39 \\
\hline Old Blush & $2 x$ & $\begin{array}{l}\text { Fen } \\
\text { Tuan } \\
\text { Qiang } \\
\text { Wei }\end{array}$ & $3 x$ & 20 & 10 & 50 & 126 & 14 & 12 \\
\hline Gold Mary & $4 x$ & $\begin{array}{l}\text { Fen } \\
\text { Tuan } \\
\text { Qiang } \\
\text { Wei }\end{array}$ & $3 x$ & 45 & 21 & 47 & 295 & 44 & 14 \\
\hline Compassion & $4 x$ & $\begin{array}{l}\text { Fen } \\
\text { Tuan } \\
\text { Qiang } \\
\text { Wei }\end{array}$ & $3 x$ & 40 & 17 & 43 & 257 & 34 & 14 \\
\hline $\begin{array}{l}\text { Carefree } \\
\text { Beauty }\end{array}$ & $4 x$ & $\begin{array}{l}\text { Fen } \\
\text { Tuan } \\
\text { Qiang } \\
\text { Wei }\end{array}$ & $3 x$ & 15 & 6 & 40 & 91 & 16 & 18 \\
\hline $\begin{array}{l}\text { Carefree } \\
\text { Beauty }\end{array}$ & $4 x$ & $\begin{array}{l}\text { Hu } \\
\text { Zhong } \\
\text { Yue }\end{array}$ & $3 x$ & 11 & 0 & 0 & - & - & - \\
\hline $\begin{array}{l}\text { Dee Dee } \\
\text { Bridgewater }\end{array}$ & $4 x$ & $\begin{array}{l}\text { Qing } \\
\text { Lian } \\
\text { Xue Shi }\end{array}$ & $3 x$ & 10 & 0 & 0 & - & - & - \\
\hline
\end{tabular}




\begin{tabular}{|llllllllll|}
\hline $\begin{array}{l}\text { Seed } \\
\text { parents }\end{array}$ & $\begin{array}{l}\text { Ploidy } \\
\text { level }\end{array}$ & $\begin{array}{l}\text { Pollen } \\
\text { parents }\end{array}$ & $\begin{array}{l}\text { Ploidy } \\
\text { level }\end{array}$ & $\begin{array}{l}\text { No. of } \\
\text { pollinated } \\
\text { flowers }\end{array}$ & $\begin{array}{l}\text { No. } \\
\text { of } \\
\text { hips }\end{array}$ & $\begin{array}{l}\text { Hip } \\
\text { set } \\
(\%)\end{array}$ & $\begin{array}{l}\text { No of } \\
\text { seeds }\end{array}$ & $\begin{array}{l}\text { No of } \\
\text { seedings }\end{array}$ & $\begin{array}{l}\text { Germina- } \\
\text { tion (\%) }\end{array}$ \\
\hline $\begin{array}{l}\text { Carefree } \\
\text { Beauty }\end{array}$ & $4 x$ & $\begin{array}{l}\text { Qing } \\
\text { Lian } \\
\text { Xue Shi }\end{array}$ & $3 x$ & 3 & 0 & 0 & - & - & - \\
$\begin{array}{l}\text { Dee Dee } \\
\text { Bridgewater }\end{array}$ & $4 x$ & $\begin{array}{l}\text { Yu Shi } \\
\text { Zhuang }\end{array}$ & $3 x$ & 21 & 7 & 33 & 168 & 50 & 30 \\
\hline $\begin{array}{l}\text { Queen } \\
\text { Elizabeth }\end{array}$ & $4 x$ & $\begin{array}{l}\text { Yu Shi } \\
\text { Zhuang }\end{array}$ & $3 x$ & 6 & 0 & 0 & - & - & - \\
\hline Total & - & - & - & 364 & 139 & 38 & 1559 & 306 & 20 \\
\hline
\end{tabular}

\section{Stigma receptivity analysis and female fertility of triploids}

'Carefree Beauty' stigmas turned to blue with high bubbling and 'Old Blush' stigmas turned yellow with high bubbling indicating high and moderate pistil receptivity respectively. In contrast, the stigmas of the five triploids showed little bubbling indicating low pistil receptivity (Fig. 4c, d, e, f, g). This agrees with the lack of set among the triploids when used as females (Table 6).

Table 6

Results of hybridization by using triploid as female parents

\begin{tabular}{|llllll|}
\hline $\begin{array}{l}\text { Seed } \\
\text { parents }\end{array}$ & $\begin{array}{l}\text { Ploidy } \\
\text { level }\end{array}$ & Pollen parents & $\begin{array}{l}\text { Ploidy } \\
\text { level }\end{array}$ & $\begin{array}{l}\text { No. of pollinated } \\
\text { flowers }\end{array}$ & $\begin{array}{c}\text { No. of } \\
\text { hips }\end{array}$ \\
\hline Chun Shui Lü Bo & $3 x$ & Chun Chao & $4 x$ & 10 & 0 \\
\hline Huzhong Yue & $3 x$ & Chun Chao & $4 x$ & 20 & 0 \\
\hline Huzhong Yue & $3 x$ & Hongwu Yue & $4 x$ & 10 & 0 \\
\hline Qinglian Xueshi & $3 x$ & Hongwu Yue & $4 x$ & 23 & 0 \\
\hline Yu Shi Zhuang & $3 x$ & Chun chao & $4 x$ & 10 & 0 \\
\hline Yu Shi Zhuang & $3 x$ & Hong wuYue & $4 x$ & 10 & 0 \\
\hline Yu Shi Zhuang & $3 x$ & $\begin{array}{l}\text { Huang shou } \\
\text { Pa }\end{array}$ & $4 x$ & 10 & 0 \\
\hline Yu Shi Zhuang & $3 x$ & $\begin{array}{l}\text { Carefree } \\
\text { Beauty }\end{array}$ & $4 x$ & 10 & 0 \\
\hline Yu Shi Zhuang & $3 x$ & Old Blush & $2 x$ & 11 & 0 \\
\hline $\begin{array}{l}\text { Fen Tuan Qiang } \\
\text { Wei }\end{array}$ & $3 x$ & Old Blush & $2 x$ & 20 & 0 \\
\hline $\begin{array}{l}\text { Fen Tuan Qiang } \\
\text { Wei }\end{array}$ & $3 x$ & $\begin{array}{l}\text { Carefree } \\
\text { Beauty }\end{array}$ & $4 x$ & 20 & 0 \\
\hline
\end{tabular}




\section{Progeny ploidy levels screening by flow cytometric analysis and chromosome counting}

Flow cytometric analyses of the 138 progenies, which were from the crosses with 'Chun Shui Lü Bo' or 'Yu Shi Zhuang' as male parents and tetraploids as female parents, indicated that 129 seedlings were tetraploid, 6 seedlings were pentaploid and 3 seedlings were triploid (Table 7 ). Chromosome counts of the pentaploid and triploid seedlings showed that these were tetraploids with 28 chromosomes (Fig. 5). Other check of the flow cytometric results with chromosome counts confirmed the ploidy designation.

Table 7

Ploidy level evaluation of rose $\mathrm{F}_{1}$ interploidy progeny by flow cytometry analysis.

\begin{tabular}{|lllllll|}
\hline Seed parents & Pollen parents & \multicolumn{3}{l}{ No. of progeny } & \multicolumn{4}{c|}{ Progeny Ploid Level } \\
\cline { 3 - 7 } & & & $\mathbf{2 x}$ & $\mathbf{3 x}$ & $\mathbf{4 x}$ & $\mathbf{5 x}$ \\
\hline Chun Chao & Chun Shui Lü Bo & 8 & 0 & 0 & 8 & 0 \\
\hline Dee Dee Bridgewater & Chun Shui Lü Bo & 56 & 0 & 0 & 56 & 0 \\
\hline Queen Elizabeth & Chun Shui Lü Bo & 17 & 0 & 1 & 13 & 2 \\
\hline Carefree Beauty & Chun Shui Lü Bo & 18 & 0 & 0 & 18 & 0 \\
\hline Tutti Frutti & Chun Shui Lü Bo & 12 & 0 & 0 & 9 & 3 \\
\hline Dee Dee Bridgewater & Yu Shi Zhuang & 28 & 0 & 2 & 25 & 1 \\
\hline Old Blush & Fen Tuan Qiang Wei & 7 & 7 & 0 & 0 & 0 \\
\hline Carefree Beauty & Fen Tuan Qiang Wei & 6 & 0 & 6 & 0 & 0 \\
\hline Compassion & Fen Tuan Qiang Wei & 17 & 0 & 17 & 0 & 0 \\
\hline Gold Mary & Fen Tuan Qiang Wei & 22 & 0 & 22 & 0 & 0 \\
\hline
\end{tabular}

Flow cytometric analyses of the 45 progenies from the crosses with 'Fen Tuan Qiang Wei' as male parents and tetraploids as female parents were all triploids, and the 7 seedlings from the cross of 'Old Blush' and 'Fen Tuan Qiang Wei' were diploid (Table 7). Chromosome counting were also conducted on 6 seedlings which were randomly selected from the cross of 'Gold Mary' and 'Fen Tuan Qiang Wei', and the results showed they were all triploid (Fig. 5), coincident with the flow cytometric analysis.

\section{Abnormal meiotic chromosome behaviors of 'Chun Shui Lü Bo' and 'Fen Tuan Qiang Wei'}

Abnormal chromosomal behaviors were recorded (Table 8) in all stages of meiosis, indicating complex chromosome pairing and unbalanced chromosome segregation in the triploid rose genotypes examined. During metaphase I and anaphase I phases, chromosomes bridges (15.8\%; Fig. 6E), lagging chromosomes (38.2\%; Fig. 6F) and chromosomes that did not align on the equatorial plate were observed (Fig. 6C, E, F, H). During meatphase II, parallel spindles (56.3\%; Fig. $6 \mathrm{~J}), 120^{\circ}$ spindles (18.8\%; Fig. $6 \mathrm{~K}$ ) and lagging 
chromosomes(16.7\%; Fig. 6L】were observed. Lagging chromosomes and unequal chromosome distributions were also observed later during anaphase II and telophase II. The most normal meiosis among the triploids was seen in 'Fen Tuan Qiang Wei', with only $29.5 \%$ of the cells in anaphase I stage showing lagging chromosomes. Unbalanced chromosome segregation and triads with two micronuclei were also observed (Fig. 7). For triploids, meiotic products were mostly tetrads with some pentads (24.2\%; Fig. 6Q), a few triads (6.7\%; Fig. 6P) as well as a few tetrads with 1 or 2 microcytes (Fig. 6R, 6T), triads with 1 or 2 microcytes (Fig. 6S)

Table 8

Meiotic abnormalities of the triploid rose 'Chun Shui Lü Bo'

\begin{tabular}{|c|c|c|c|c|}
\hline Phases & $\begin{array}{l}\text { Number of } \\
\text { observed } \\
\text { cells }\end{array}$ & Abnormalities & $\begin{array}{l}\text { No. of } \\
\text { abnormal cells }\end{array}$ & $\begin{array}{l}\text { Abnormal } \\
\text { cells (\%) }\end{array}$ \\
\hline 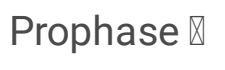 & 254 & - & 0 & 0 \\
\hline $\begin{array}{l}\text { Metaphase } \\
\square\end{array}$ & 2216 & Lagging chromosome & 165 & $7.5 \%$ \\
\hline \multirow[t]{2}{*}{ Anaphase $\rrbracket$} & \multirow[t]{2}{*}{424} & \multirow[t]{2}{*}{ Lagging chromosome Bridge } & 162 & $38.2 \%$ \\
\hline & & & 67 & $15.8 \%$ \\
\hline Telophase & 84 & $\begin{array}{l}\text { Chromosome not align on the } \\
\text { equatorial plate }\end{array}$ & 18 & $21.4 \%$ \\
\hline \multirow{3}{*}{$\begin{array}{l}\text { Metaphase } \\
\text { II }\end{array}$} & \multirow[t]{3}{*}{48} & Parallel spindles & 27 & $56.3 \%$ \\
\hline & & $120^{\circ}$ spindles & 9 & $18.8 \%$ \\
\hline & & Lagging chromosome & 8 & $16.7 \%$ \\
\hline \multirow{2}{*}{$\begin{array}{l}\text { Anaphase } \\
\text { II }\end{array}$} & \multirow[t]{2}{*}{16} & Lagging chromosome & 4 & $25.0 \%$ \\
\hline & & Unequal distribution & 1 & $6.3 \%$ \\
\hline $\begin{array}{l}\text { Telophase } \\
\text { ॥I }\end{array}$ & 43 & Lagging chromosome & 1 & $2.3 \%$ \\
\hline \multirow{7}{*}{$\begin{array}{l}\text { Meiotic } \\
\text { product }\end{array}$} & \multirow[t]{7}{*}{1429} & Triad & 95 & $6.7 \%$ \\
\hline & & Triads with microcytes & 18 & $1.3 \%$ \\
\hline & & Triads with two microcytes & 6 & $0.4 \%$ \\
\hline & & Tetrads with microcytes & 150 & $10.5 \%$ \\
\hline & & Tetrads with 2 microcytes & 6 & $0.4 \%$ \\
\hline & & Penads & 346 & $24.2 \%$ \\
\hline & & Penads with microcytes & 28 & $2.0 \%$ \\
\hline
\end{tabular}


Table 9

Meiotic abnormalities of triploid 'Fen Tuan Qiang Wei'

\begin{tabular}{|llll|}
\hline Phases & $\begin{array}{l}\text { Number of observed } \\
\text { cells }\end{array}$ & Abnormalities & Abnormal cells (\%) \\
\hline Anaphase $\otimes$ & 536 & Lagging chromosome & $29.5 \%$ \\
\hline Meiotic product & 610 & Triads & $12.5 \%$ \\
\hline
\end{tabular}

\section{Discussion}

The positive correlation between DNA content and pollen size makes it possible to decide the ploidy level by measuring the pollen size (Dewitte et al. 2012). For normal diploid plants, the frequency distribution of pollen grain size is unimodal, but for unreduced pollen producers, it is bimodal (Tondini et al.1993). In this research, pollen grains size of 'Old Blush'(diploid) 'Dee Dee Bridgewater' (tetraploid) and 'Fen Tuan Qiang Wei' (triploid) followed a unimodal distribution, suggesting that 1 ploidy level pollen grain were produced. For triploid 'Chun Shui Lü Bo' and 'Yu Shi Zhuang', the size of pollen grains had higher variation coefficient (from $18.8 \%$ to $24.3 \%$ ) and followed a skewed, flattened, and slightly bimodal distributions, suggesting the wide range of pollen size in triploid, which may represent the various chromosome numbers of pollen grains due to irregular chromosome pairing and unbalanced chromosome segregation.

For the 5 triploid China rose cultivars used as pollen parents in this study, 2 of them did not set any hips, 3 of them gave good hip and seed production on the tetraploid mother plant. This result indicated that some of the triploid China Rosa germplasm do have good fertility ability, and can be used as gene introgression bridge in future rose breeding program.

In this study, when triploid 'Fen Tuan Qiang Wei' was used as male parent, it produced $1 \mathrm{n}$ pollen as indicated by the unimodal pollen size distribution, the triploid seedlings from the $4 x(\mathbb{x}) \times 3 x(\mathbb{Q})$ crosses, and the diploid seedlings from the $2 x(\mathbb{X}) \times 3 x(\mathbb{X})$ crosses. This result is different from Leus's, Zlesak's and Barden's work (Leus, 2005; Zlesak, 2009; Barden and Zlesak, 2004), and also different from the crosses in current study when 'Chun Shui Lü Bo' or 'Yu Shi Zhuang' were used as male parents. This is the first report that triploid rose producing mostly $1 \mathrm{n}$ viable pollen, and $1 \mathrm{n}$ pollen is more vigor than $2 \mathrm{n}$ pollen. It is a special result. To my knowledge, the only similar report I can find is about Hieraciumechioides, Peckert reported he got about $80 \%$ triploids in $4 x \times 3 x$ crosses (Peckert, 2006).

When 'Chun Shui Lü Bo' and 'Yu Shi Zhuang' were used as male parents and crossed with a tetraploid female, $2 \mathrm{n}$ pollen grains were favored for fertilization as all of the seedlings from these crosses are tetraploid. This result is similar to Leus's report, he (2005) obtained about $98 \%(123 / 125)$ tetraploid offspring

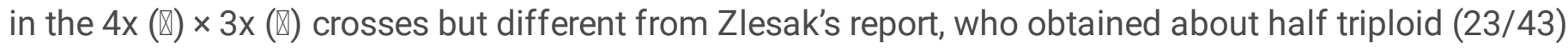
offspring and half tetraploid offspring $(20 / 43)$ in the $4 x(\nabla) \times 3 x(\nabla)$ crosses. Greater vigor of $2 n$ pollen was the reason for most offspring were tetraploid in this research and in Leus's research.

In current study, when triploids were used as male parents, only euploid progeny were obtained, the same as Leus's and Zlesak's work. It seems anueploid progeny are rarely produced when triploid Rosa are used as 
male parents. This is different from the studies about apple (Zhang, 2009), lily (Zhang, 2017) and populus (Wang, 2017), in which more aneuploid than euploid progeny were obtained. This is also different from Ramsey's report. In Ramsey's summary, the most common pollen chromosome number was $3 x / 2$, followed by $3 \times / 2-1$, and the lowest pollen chromosome number was $1 \times(3 \%)$ and diploid $2 \times(2 \%)$ (Ramsey, 1998). In current study, the wide range of pollen size of 'Chun Shui Lü Bo' and 'Yu Shi Zhuang' suggested the various chromosome numbers of pollen grains. So probably some of the pollens were anueploid. The reason why all the progeny obtained in this study were euploid may be that euploid gametes have greater vigor than the aneuploidy gametes in fertility. Though the aneuploidy pollen is not vigorous, in crosses with triploid Rosa as male parents, the fruit set and the average number of seeds per hip were higher compared with most other triploids (Zhang, 2017; Zhang, 2009; Wang, 2017). This result indicates that the Rosa triploids have some kind of mechanism to make sure they can produce much more vigorous euploid progeny than other triploids.

In this research, the triads, pentads and microcyte in the meiosis products indicated the objective triploid production of euploid pollen grains. Microcyte formation likely resulted in the loss of chromosomes from microspores, which may increase the variation in pollen size. According to Ramsey, euploid gametes are very rare, because haploid-diploid chromosome assortments are only produced when the separation of multivalents and unpaired chromosomes at the first meiotic division in a spore mother cell is very unequal (Ramsey and Schemske1998). Few studies had been done about the unique mechanism of meiosis in triploid roses. The reasons why triploid roses can produce so many euploid gametes, and why 'Fen Tuan Qiang Wei' produced $1 \mathrm{n}$ viable pollen whereas 'Chun Shui Lü Bo' and 'Yu Shi Zhuang' produced $2 \mathrm{n}$ viable pollen are still uncertain. More researches need to be done to clarify the mechanism in the future.

\section{Conclusion}

In this study we reported 1 China triploid rose produces $1 \mathrm{n}$ viable pollen, and 2 China triploid roses produce $2 \mathrm{n}$ viable pollen, and no anueploid progeny was produced. Our results indicate that the triploids contribute significantly to tetraploids formation in the crossing system of Genus Rosa, and it is possible for breeders to create novel rose types with valuable triploids in the future. With the special triploid resources found in this experiment, Genus Rosa will be an excellent material to study the mechanism of triploids in the future.

\section{Abbreviations}

Not applicable.

\section{Declarations}

Ethics approval and consent to participate: Not applicable.

Consent for publication: Not applicable.

Availability of data and material: All the data can be available, and it is according to China law that if the material can be available. 
Competing interests: This research does not have any conflicts of interest.

Funding: This research was funded by Beijing Municipal Science \& Technology Commission (z191100008519005) and Beijing Municipal Administration Center of Parks (2017076). There is no role of the funding body in the design of the study and collection, analysis, and interpretation of data and in writing the manuscript.

Authors' contributions: SWZ and HF developed the idea for this study, NZJ, YX, BBG and YHB performed the research, HF wrote this paper. All authors read and agree this paper.

Acknowledgments: The author would like to thank Dr. David Byrne at Texas A\&M University for his valuable comments and constructive suggestions to this paper.

\section{References}

1. Barden P, Zlesak DC. Conducting chromosome counts on the Bracteata hybrids. Rosa Hybridizers Association Newsletter. 2004;35(4):12-7.

2. Blanc $\mathrm{G}$, Wolfe $\mathrm{KH}$. Wide spread paleo polyploidy in model plant species inferred from age distributions of duplicate genes. Plant Cell. 2004;16:1667-78.

3. Burton TL, Husband BC. Fecundity and offspring ploidy in matings among diploid, triploid and tetraploid Chamerion angustifolium (Onagraceae): consequences for tetraploid establishment. Heredity. 2001;87:573-82.

4. Crespel L, Ricci SC, Gudin S. The production of 2n pollen in rose. Euphytica. 2006;151:155-64.

5. Del Bosco SF, Tusa N, Conicella C. Microsporogenesis in a Citrus interspecific tetraploid somatic hybrid and its fusion parents. Heredity. 1999;83:373-7.

6. Dewitte A, Van Laere K, Van Huylenbroeck J. Use of 2ngametes in plant breeding. In: Abdurakhmonov IY, editor. Plant breeding. Croatia: InTech Open Access Publisher; 2012. pp. 59-86. doi:10.5772/29827.

7. Du YP, Wei C, Wang ZX, Li S, He HB, Jia GX. Lilium spp. pollen in China (Liliaceae): taxonomic and phylogenetic implications and pollen evolution related to environmental conditions. PLoS One. 2014;9:1-19.

8. Erdtman G. Pollen Morphology and Plant Taxonomy of Angiosperms. Angiosperms. New York: Hafner Publishing Co; 1971.

9. Feng $\mathrm{H}$, Wang ML, Cong RC, Dai SL. Colchicine- and trifluralin-mediated polyploidization of rosa multiflora thunb. var. inermis and rosa roxburghii f. normalis. The Journal of Horticultural Science Biotechnology. 2017;92:279-87.

10. Husband BC. The role of triploid hybrids in the evolutionary dynamics of mixed-ploidy populations. Biol J Linn Soc. 2004;82:537-46.

11. Jacob Y, Pierret V. Pollen size and polidy level in the genus. Rosa Acta Horticulture. 2000;508:289-92.

12. Kevan PG. Pollination. In: Roberts AV, Debener T, Gudin S, editors. Encyclopedia of Rose Science. (Vol 1). Oxford: Elsevier Academic Press; 2003. pp. 456-60. 
13. Kho YO, Bera J. Observing pollen tubes by means of fluorescence. Euphytica. 1968;17:299-302.

14. Leus L. (2005) Resistance breeding for powdery mildew (Podosphaera pannosa) and black spot (Diplocarpon rosae) in roses. PHD thesis, Ghent University, Belgium.

15. Brito MS, Ligia T, Bertolino V, Cossalter AC, Quiapim, et al. Pollination triggers female gametophyte development in immature Nicotiana tabacum flowers. Front Plant Sci. 2015;6:561.

16. Monika A, Makwana, Parihar A. Stigma receptivity test in diverse species of tomato. Intermational Journal of Agricultural Scienc Research. 2017;7(5):1-8.

17. Peckert T, Jun JC. Mating interactions between coexisting diploid, triploid and tetraploid cytotypes of Hieraciumechioides (Asteraceae). Folia Geobotanica. 2006;41(3):323-34.

18. Risso-Pascotto C, Pagliarini MS, Do Valle CB. Multiple spindles and cellularization during microsporogenesis in an artificially induced tetraploid accession of Brachiariaruziziensis (Gramineae). Plant Cell Rep. 2005;23:522-7.

19. Ramsey J, Schemske DW. Pathways, mechanisms, and rates of polyploid formation in flowering plants. Annu Rev Ecol Syst. 1998;29:467-501.

20. Simillion C, Vandepoele K, Van Montagu MCE, Zabeau M, Van de Peer Y. (2002) The hidden duplication past of Arabidopsis thaliana. Proc. Natl. Acad.Sci. 99: 13627-13632.

21. Tondini F, Tavoletti S, Mariani A, Veronesi F. A statistical approach to estimate the frequency of $n, 2 n$ and 4n pollen grains in diploid alfalfa. Euphytica. 1993;69:109-14.

22. Van Tuyl JM, Lim K-B. Interspecific hybridisation and polyploidisation as tools in ornamental plant breeding. Acta Hort. 2003;612:13-23.

23. Wang J, Huo B, Liu W, Li D, Liao L. (2017) Abnormal meiosis in an intersectional allotriploid of Populus L. and segregation of ploidy levels in $2 x \times 3 x$ progeny. PLOS ONE $12(7)$.

24. Wang J, Kang XY, Zhu Q. Variation in pollen formation and its cytological mechanism in an allotriploid white poplar. Tree Genet Genomes. 2010;6:281-90.

25. Wang J, You HL, Tian J, Wang YF, Liu MH, Duan WL. Abnormal meiotic chromosome behavior and gametic variation induced by intersectional hybridization in Populus L. Tree Genet Genomes. 2015;11:61.

26. Zhang $\mathrm{CH}$, Park SM. Aneuploid production from crosses with diploid and triploid in apple tree. Horticulture Environment Biotechnology. 2009;50(3):203-7.

27. Zhang X, Cao Q, Zhou P, Jia G. Meiotic chromosome behavior of the male-fertile allotriploid lily cultivar 'Cocossa'. Plant Cell Rep. 2017;36(10):1641-53.

28. Zlesak DC. (2009). Pollen diameter and guard cell length as predictors of ploidy in diverse rose cultivars, species, and breeding lines. In: Zlesak DC, editor Roses. Floriculture and Ornamental Biotechnology. 3(Special Issue 1):53-70.

\section{Figures}



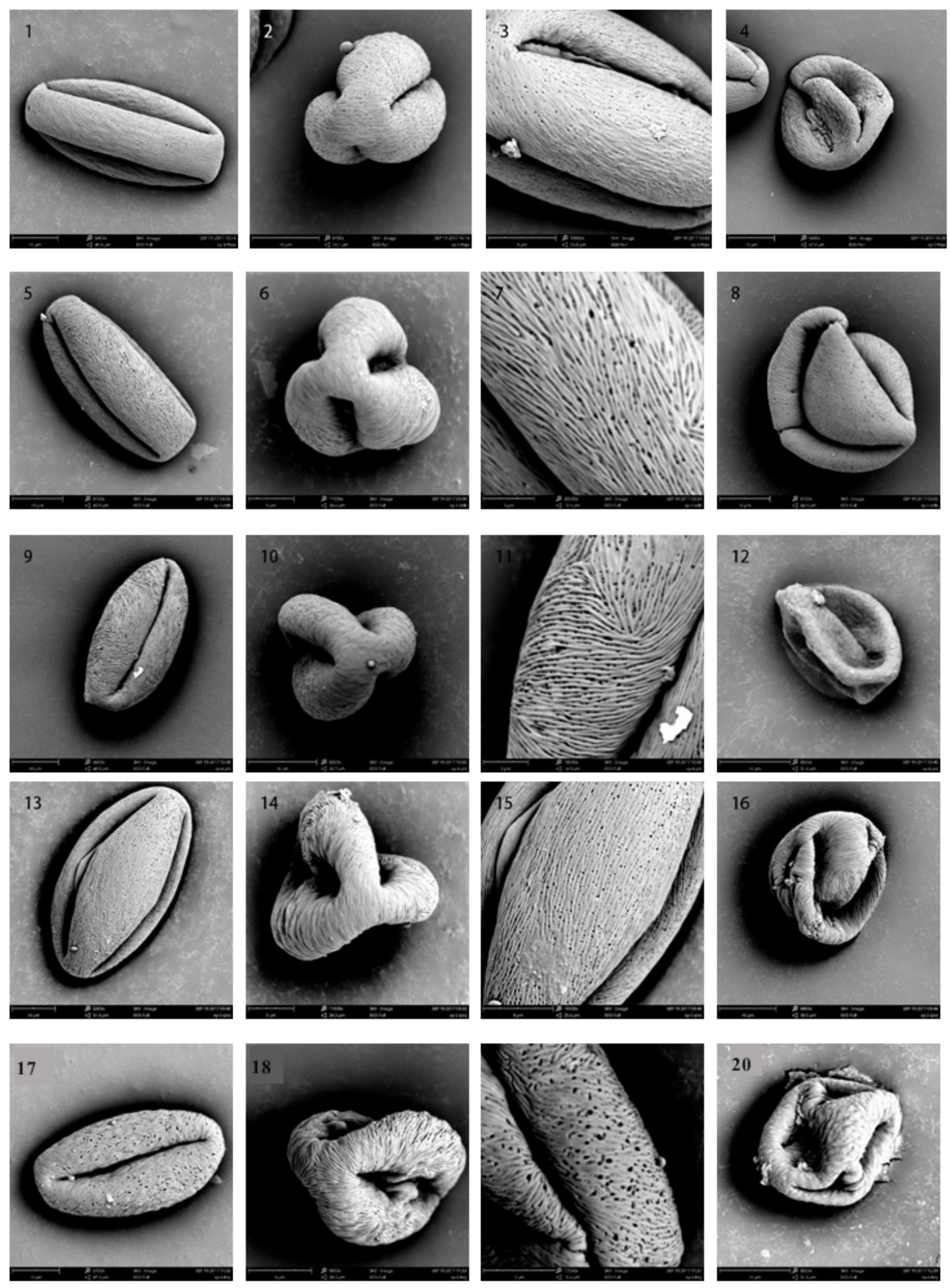

Figure 1

Scanning electron micrographs of pollen grains from five triploid roses in polar and equatorial view. 1-4, Polar view, equatorial view, external surface, and anomalous pollens of 'Fen Tuan Qiang Wei'; 5-8, Polar view, equatorial view, external surface, and anomalous pollens of 'Chun Shui Lü Bo'; 9-12, Polar view, equatorial view, external surface, and anomalous pollens of 'Yu Shi Zhuang';13-16, Polar view, equatorial view, external surface, and anomalous pollens of 'Qing Lian Xue Sshi'; 17-20, Polar view, equatorial view, external surface, and anomalous pollens of 'Hu Zhong Yue'. 

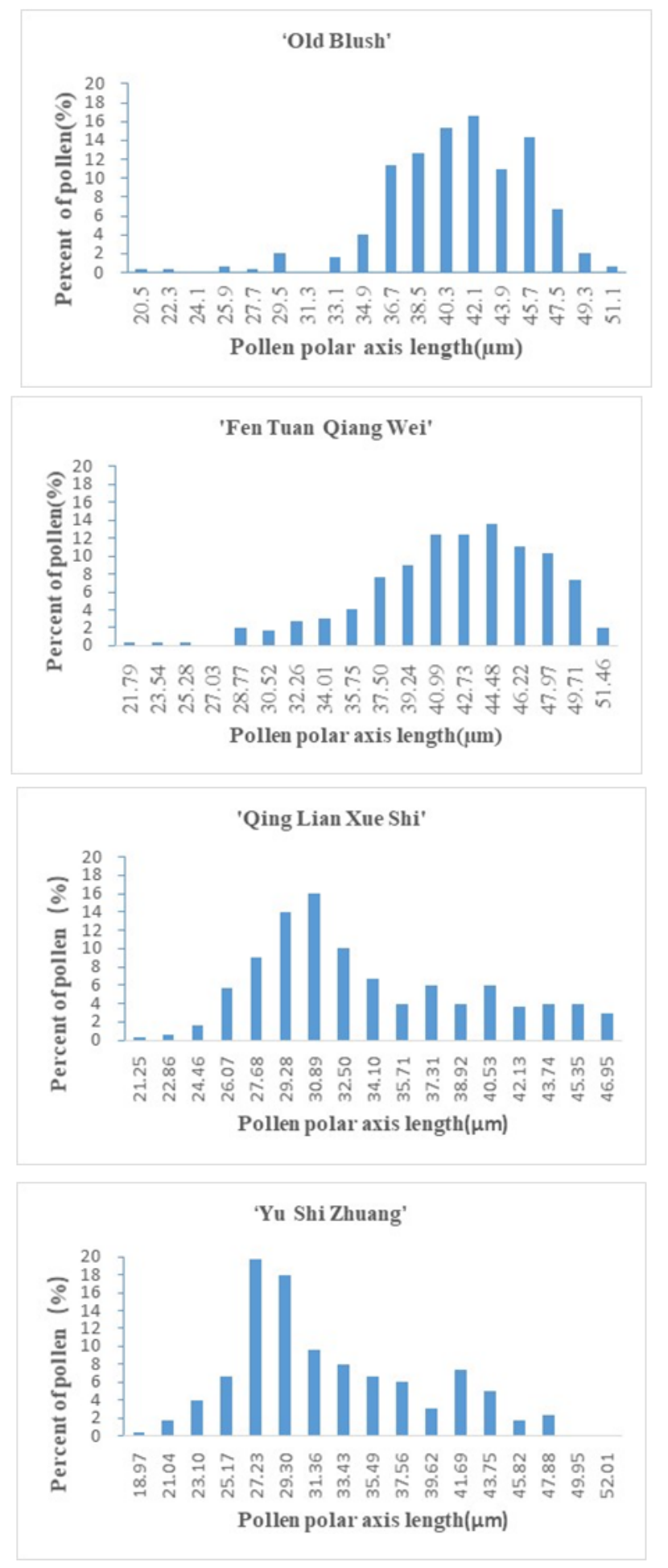
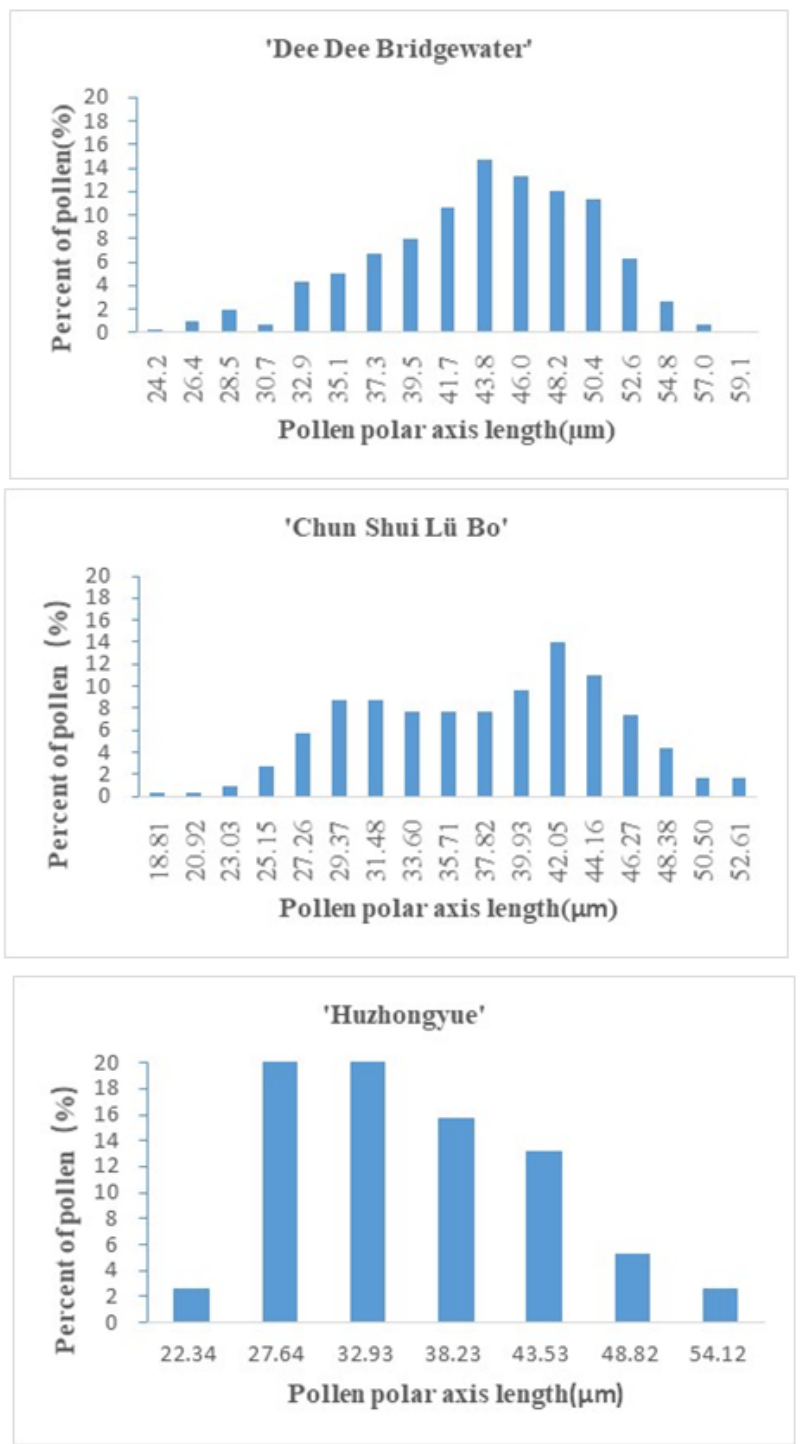

\section{Figure 2}

Frequency distribution with polar axis length of pollen grains from five triploid roses, 'Old Blush' (diploid) and 'Dee Dee Bridgewater' (tetraploid). 

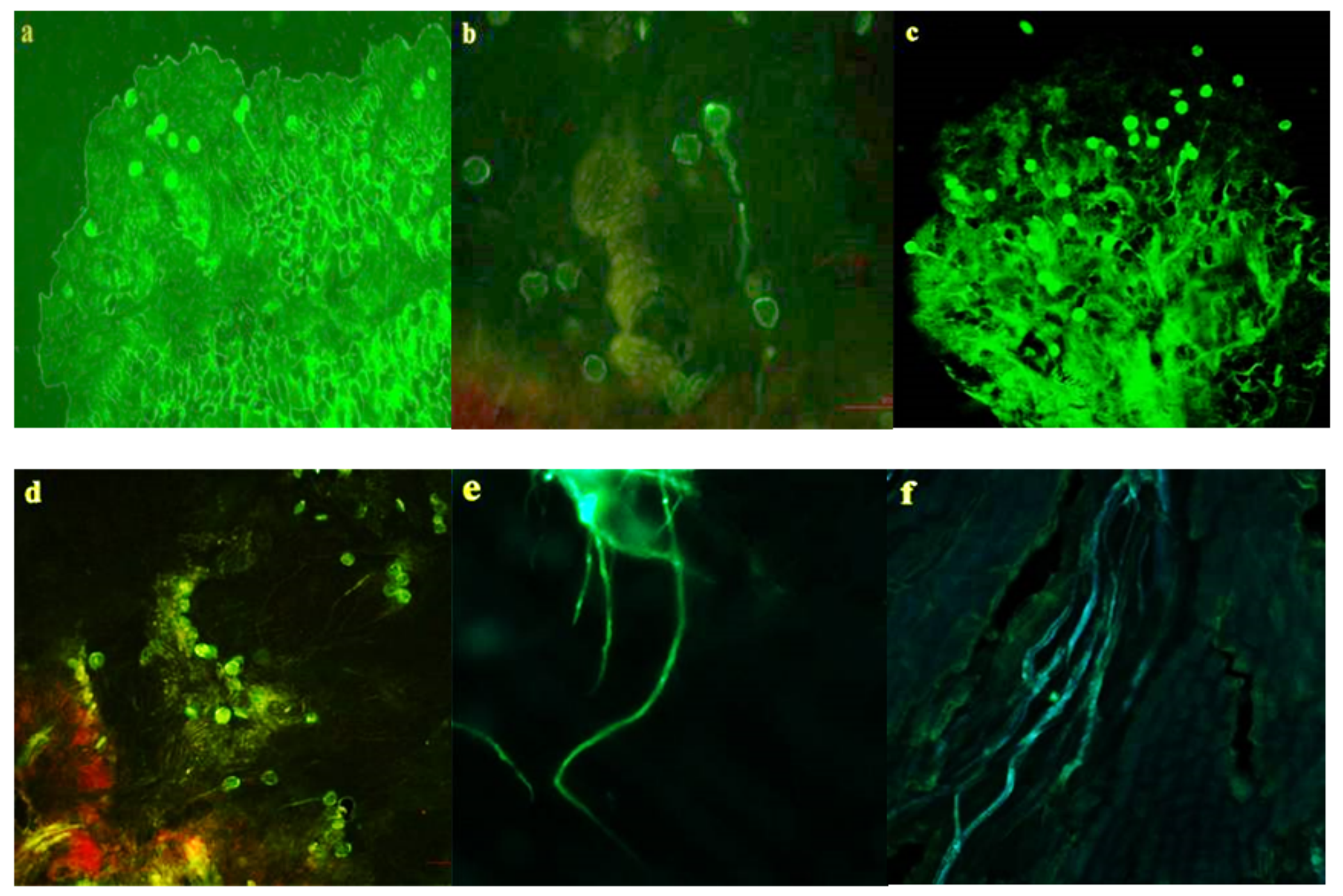

\section{Figure 3}

Pollens from 'Chun Shui Lü Bo' germination and pollen tube growth in pistils of 'Carefree Beauty', viewed using fluorescence microscopy. a. Germinating pollen grains on surface of stigma, $8 \mathrm{~h}$ after cross-pollination

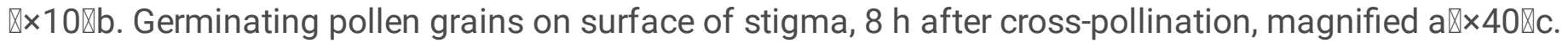
Pollen tube elongation, $12 \mathrm{~h}$ after pollination $\nabla \times 20 \bigotimes \mathrm{d}$. Pollen tube reaching upper part of style, $24 \mathrm{~h}$ after pollination $\nabla \times 20 \rrbracket$ e. Pollen tube reaching lower part of style, $48 \mathrm{~h}$ after pollination $\nabla \times 40 \nabla f$. Pollen tube entering ovules, $72 \mathrm{~h}$ after pollination $₫ \times 40 \bigotimes$. 

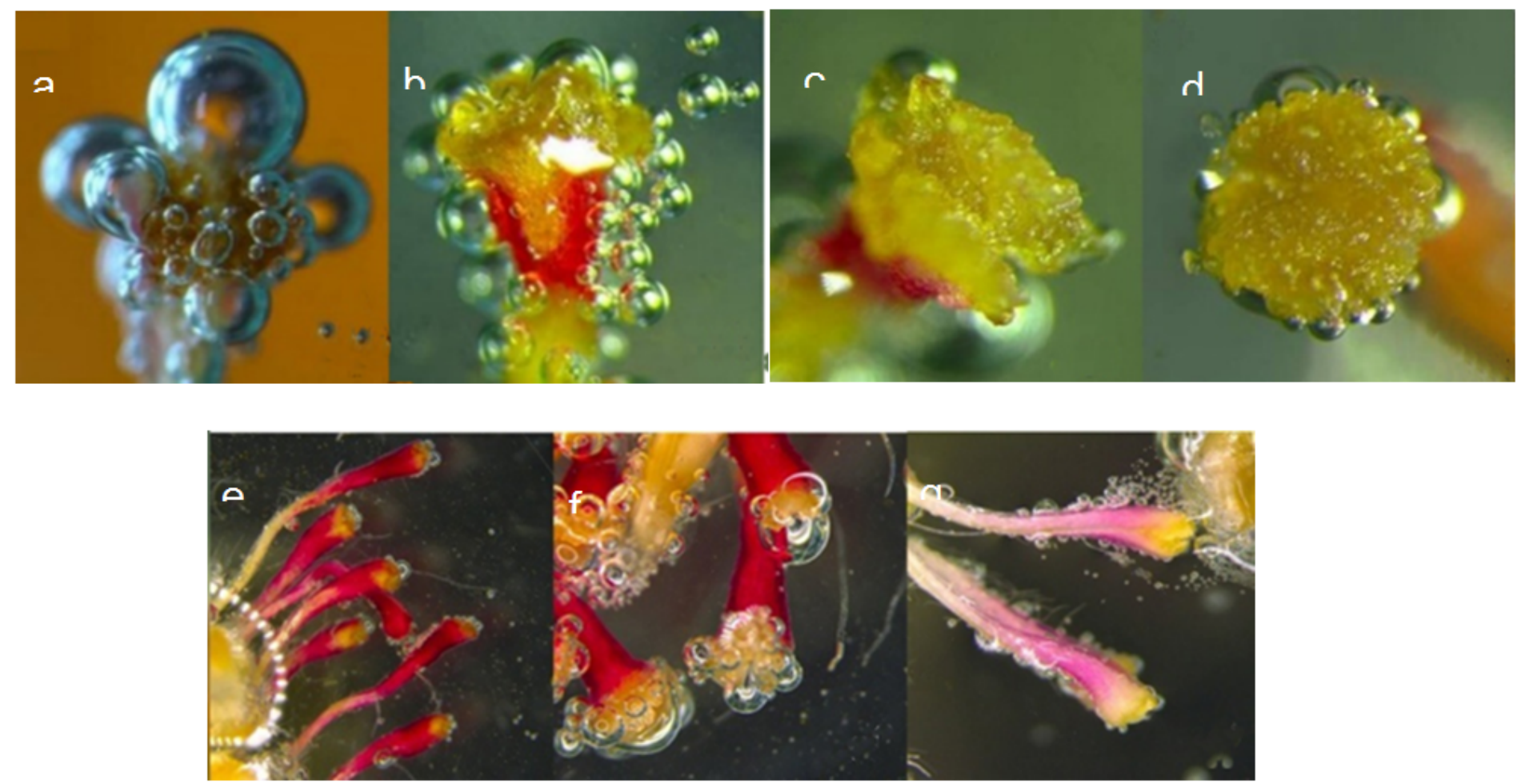

\section{Figure 4}

Stigma receptivity of five triploid roses, 'Old Blush' (diploid) and 'Carefree Beauty' (tetraploid). a: 'Carefree Beauty' ; b: 'Old Blush'; c: 'Fen Tuan Qiang Wei'; d: 'Chun Shui Lü Bo'囚e: 'Hu Zhong Yue'; f: 'Qing Lian Xue Shi'; g: 'Yu Shi Zhuang'. 


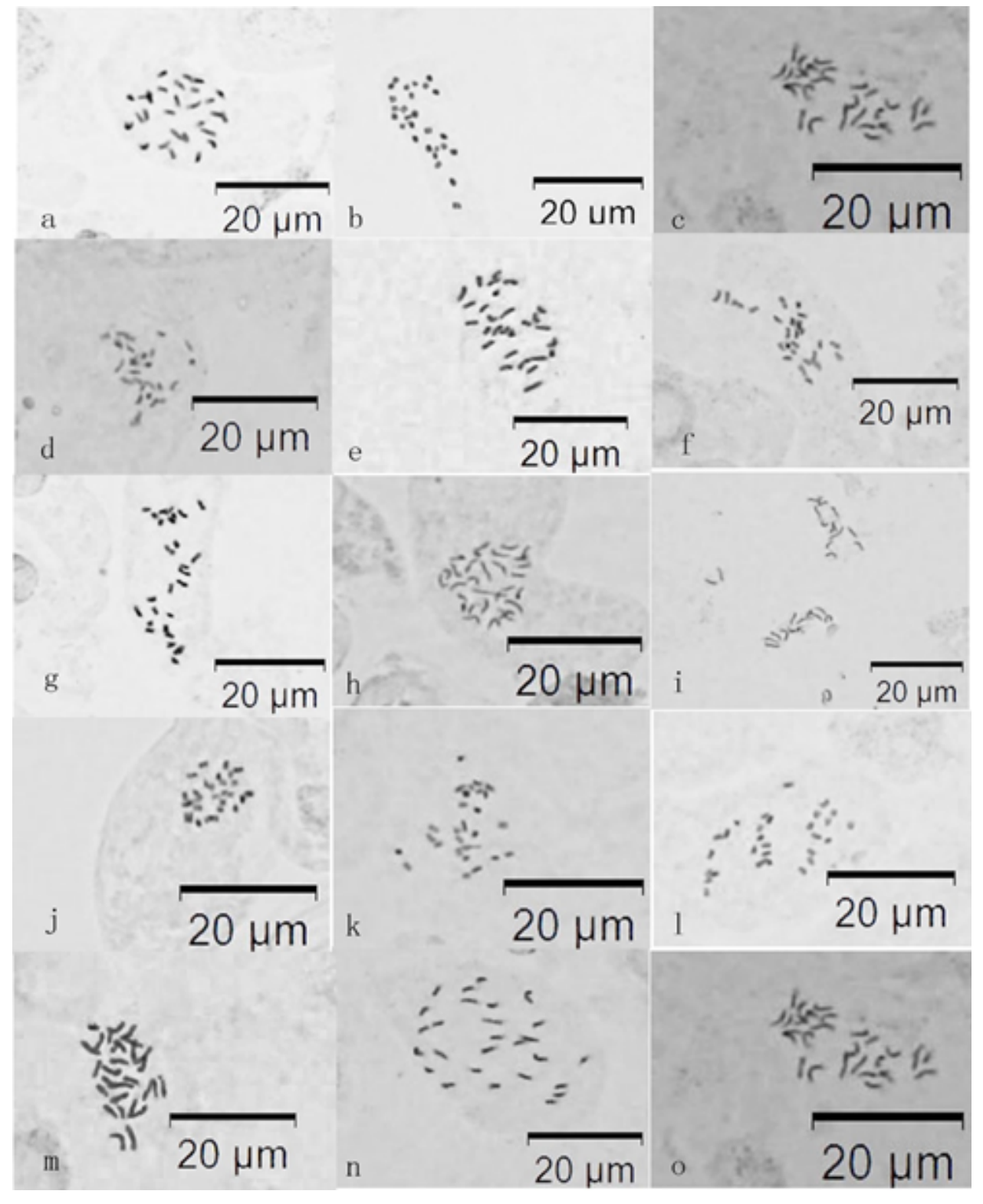

\section{Figure 5}

Mitotic chromosome numbers in root tip cells from interploidy crosses with triploids as male parents, a-g

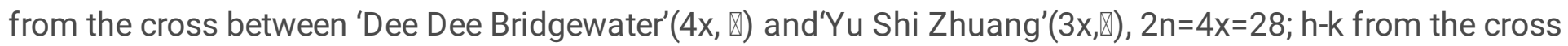

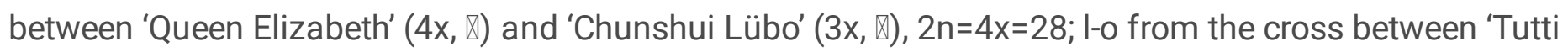

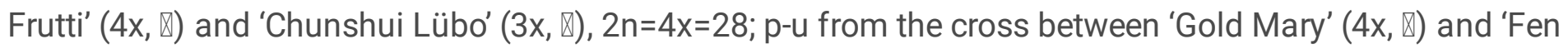
Tuan Qiang Wei' $(3 x, \mathbb{x}), 2 n=3 x=21$. 

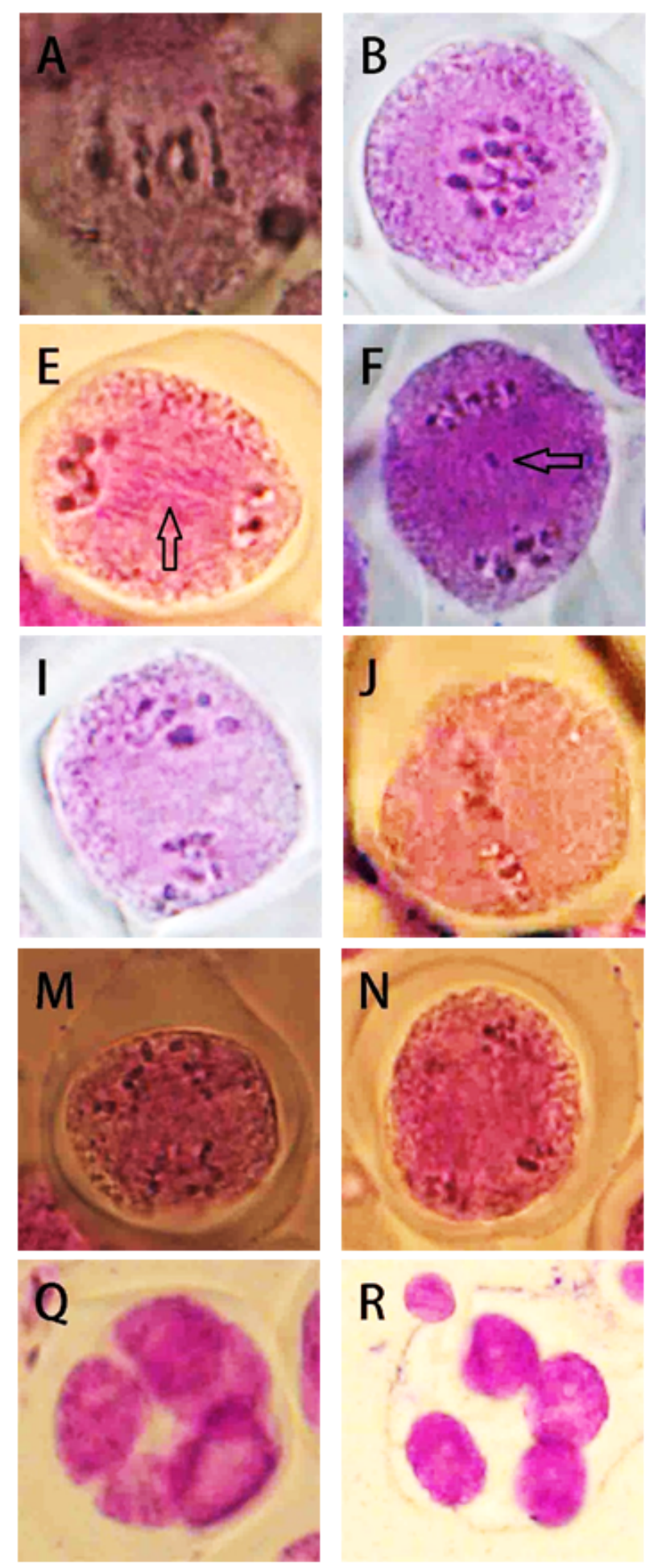
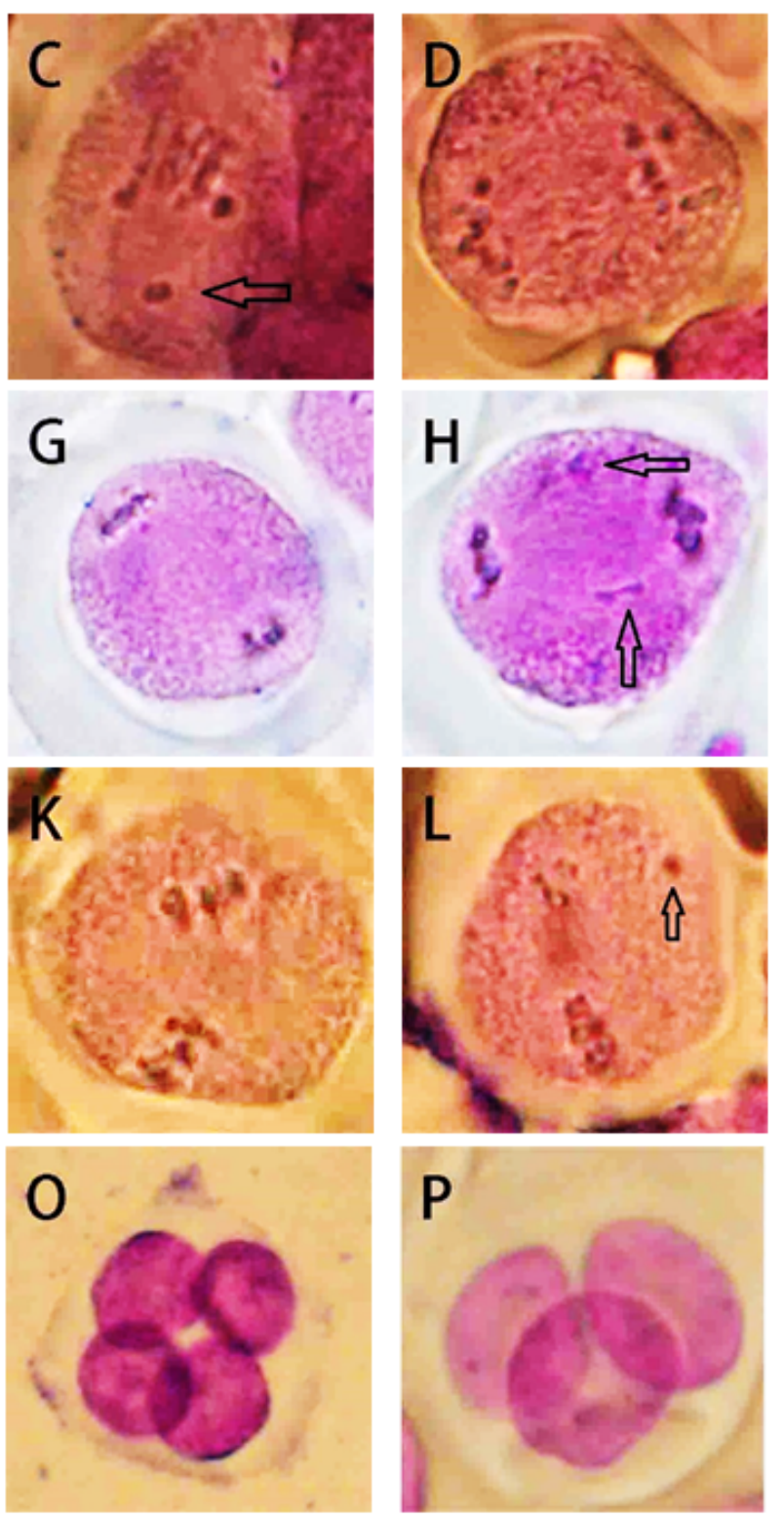

S
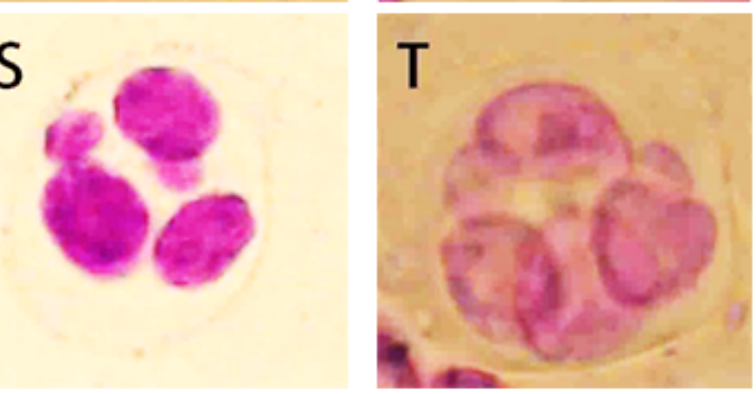

\section{Figure 6}

Meiosis chromosomal behavior of 'Chun Shui Lü Bo' pollen mother cells A-B metaphase $\rrbracket, C$ metaphase $\rrbracket$

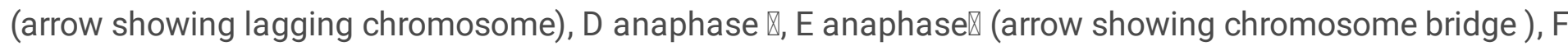

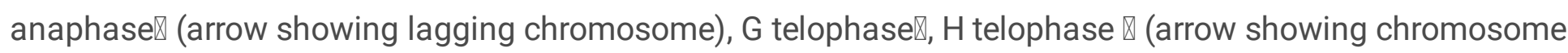

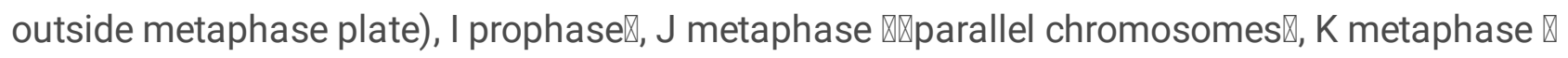

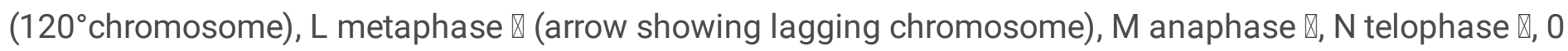


tetrad, P triad, Q quartet, R quadrant with microspores, S Triad with two microcytes, T quadrant with two microcytes.

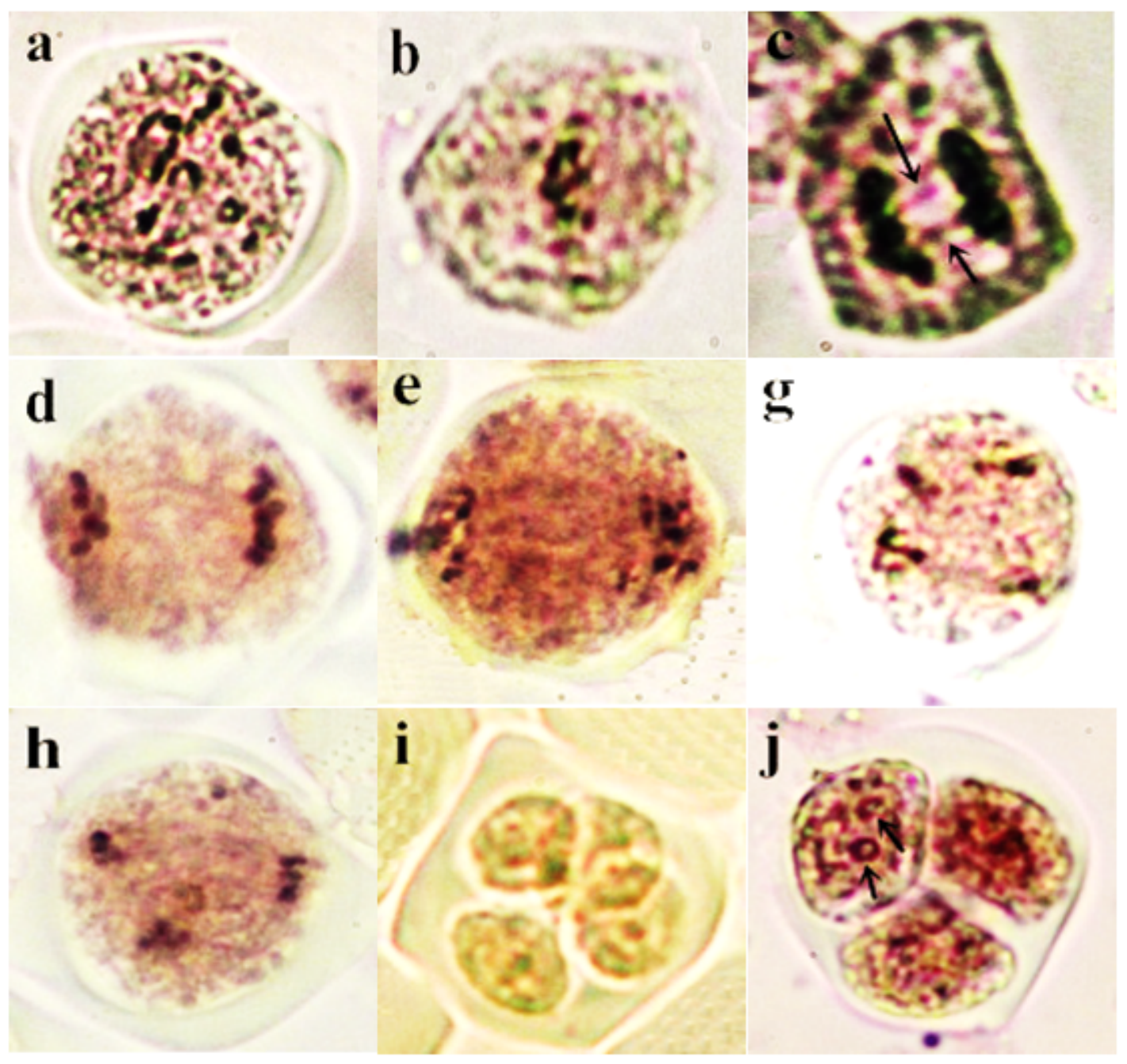

Figure 7

Meiosis chromosomal behavior of R. multiflora 'Fen Tuan Qiang Wei' pollen mother cells, a Prophase区, b

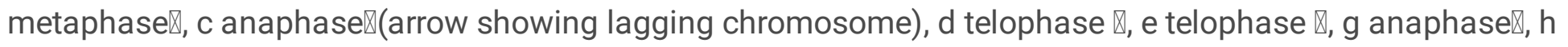
tripolar spindles at telophase $\Downarrow$, showing 3 nuclei were produced, $\mathrm{i}$ tetrads, j triads with 2 micronuclei 